\title{
Improving The Manoeuvrability of Electric Vehicle with Four-Wheel Drive and Four-Wheel Steering - A Nonlinear Model Vehicle Dynamics Approach
}

\author{
Ishak M.I ${ }^{1 *}$, Heerwan P.M ${ }^{1}$, and Rasid M.A.H ${ }^{1}$ \\ ${ }^{1}$ Faculty of mechanical engineering, Universiti Malaysia Pahang, Pekan Campus, 26600 Pekan, \\ Pahang, Malaysia
}

\begin{abstract}
The dynamics motion of a vehicle is inherently a nonlinear dynamics system especially at high speed. Majority of past researches on four-wheel steering (4WS) vehicle adopt easier way of modelling a control system based on vehicle with linear dynamic equation of motion. This paper study on the vehicle dynamics of an electric vehicle with 4WD and 4WS based on nonlinear vehicle dynamic approach. A numerical simulation was performed to analyse the variance of a linear model and nonlinear model during cornering at various constant speed. The results show that during low speed cornering at $10 \mathrm{~km} / \mathrm{h}$, the linear and nonlinear model produced similar steady state cornering based on the trajectory and yaw rotational speed. However, the variants of linear and nonlinear started to appear as the vehicle speed increase. By obtaining the steady state cornering speed, another numerical simulation was performed to analyse the characteristics of the 4WD and 4WS electric vehicle. A passive control of the rear wheels' steer angle was implement in the simulation. The results show that the parallel steering mode decreased the yaw rotational speed which broaden the trajectory of the cornering, while the opposite steering mode increased the yaw rotational speed that led to a tighter trajectory during cornering.
\end{abstract}

\section{Introduction}

As the automotive industry progress in the usage of electric based transportation, demands on the stability, controllability, and feasibility of electric vehicle simultaneously increase. Some of the mechanical link schema of conventional vehicle will be converted into electric modules which only require signal cables. By combining electric powered and by-wire control technology, major mechanical part can be downsized and allow freedom of layouts and driving potentials. Due to this reason, four-wheel drive (4WD) and four-wheel steering (4WS) system for electric vehicle is much more accomplishable compare to conventional vehicle.

\footnotetext{
*Corresponding author: mizhar@ump.edu.my
} 
The dynamics motion of a vehicle is inherently a nonlinear system especially at high speed. It is highly potential that a front wheel steering (FWS) vehicle neutral steer at low speed and oversteer or understeer at high speed. A 4WS vehicle with stability control technology makes it possible for a commercial vehicle to steer even at high speed. However, much of past researches on four-wheel steering (4WS) vehicle adopt easier way of modelling a control system based on vehicle with linear dynamic equation of motion [1-5]. During low speed, the 4WS control system can be considered acceptable. However, when assuming a $4 \mathrm{WS}$ vehicle as a linear system at high speed, other parameters that could influence the dynamic motion of the vehicle were neglected and thus producing a control model that is unsuitable to the real vehicle.

In this paper, a study on the vehicle dynamics of an electric vehicle with 4WD and 4WS based on nonlinear model was done. Firstly, a numerical simulation was performed to analyse the variance of a linear model and nonlinear model of an electric vehicle with four-wheel drive and four wheel steering during cornering at various constant speed and at a constant front wheel steer angle. Variants of linear and nonlinear started to appear as the vehicle speed increase.

By obtaining the steady state cornering speed, another numerical simulation was performed to analyse the characteristics of the 4WD and 4WS electric vehicle during cornering at constant speed and at a constant front wheel steer angle. A passive control of the rear wheels' steer angle was implement in the simulation.

\section{Main Symbols}

$b$ : width of tire interacted surface $=0.1 \mathrm{~m}, d_{F}$ : front tread $=0.84 \mathrm{~m}, d_{R}$ : rear tread $=0.815$ $\mathrm{m}, I$ : yaw inertia moment at gravity point of vehicle $=1470 \mathrm{kgm}^{2}, K_{x}, K_{y}:$ tire stiffness $=$ $3.33 \times 10^{6} \mathrm{~N} / \mathrm{m}^{3}, k_{F}, k_{R}$ : front and rear tire cornering stiffness, $l$ : length of tire interacted surface $=0.15 \mathrm{~m}, l_{F}$ : length from front axle to gravity point of vehicle $=0.64 \mathrm{~m}, l_{R}$ : length from rear axle to gravity point of vehicle $=0.64 \mathrm{~m}, m$ : mass of the vehicle $=421.61 \mathrm{~kg}, u$ : longitude velocity, $v$ : lateral velocity, $X_{F R,} X_{F L}, X_{R R}, X_{R L}$ : friction force for each tire, $Y_{F R}, Y_{F L}, Y_{R R}, Y_{R L}$, lateral force for each tire, $W_{z}$ : wheels load subject to load transfer, $\beta$ : side slip angle of nonlinear model, $\beta^{*}$ : side slip angle of linear model, $\beta_{F R}, \beta_{F L}, \beta_{R R}, \beta_{R L}$ : tire side slip angle, $\mu$ : friction coefficient, $\theta_{F}, \theta_{R}$ :front and rear wheels steer angle, $\gamma$ : yaw rotational speed of vehicle body, $\gamma^{*}$ : yaw rotational speed of linear model, $\rho$ : slip ratio, $\omega$ : tire rotational speed.

\section{Vehicle Model}

\subsection{Vehicle model}

The specifications of the Toyota COMS EV (Figure 1) is used as a test model in the simulation. Although the vehicle is a 2WD in-wheel EV and has only mechanical front-wheel steering, it is configured in simulations with a four-wheel drive and four-wheel steering system. The weight of the rear wheel in-wheel motor was calculated and added to the front of the vehicle.

\subsection{Linear Dynamic Equation of Motion}

Commonly, we considered that there is no difference in the characteristics of the left and right tires due to linearity. Therefore, a two-wheel model is derived from the equations. If the side-slip angle is small, it is assumed that the direction perpendicular to the traveling direction 
of the vehicle almost coincides with the lateral direction $y$. Therefore, no coupling exists between the yaw rotational speed and the vehicle's translational velocity which makes the linear dynamic equation of motion [6].

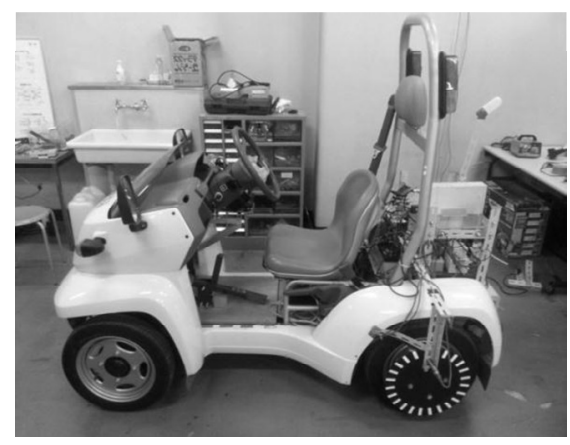

Fig.1 Toyota COMS EV

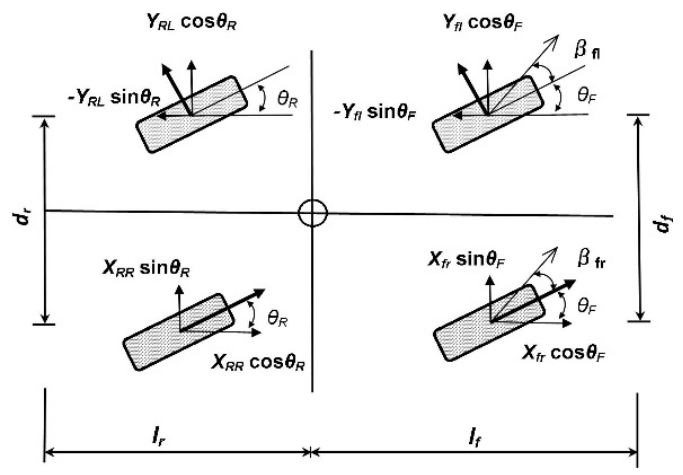

Fig.2 Force vector for four wheel steering vehicle

$$
\begin{gathered}
\mathrm{m} V\left(\frac{d \beta^{*}}{d t}+\gamma^{*}\right)=2 Y_{f}+2 Y_{r}+G_{s} \\
\mathrm{I} \frac{d \gamma^{*}}{d t}=\left(2 l_{f} Y_{f}-2 l_{r} Y_{r}-l_{s} G_{s}\right) \cos \beta^{*}
\end{gathered}
$$

The linear lateral force for a four-wheel steering is defined by;

$$
\begin{aligned}
& Y_{f}=-K_{f} \beta_{f}=-K_{f}\left(\beta^{*}+\frac{l_{f}}{v} \gamma^{*}-\theta_{f}\right) \\
& Y_{r}=-K_{r} \beta_{r}=-K_{r}\left(\beta^{*}-\frac{l_{r}}{v} \gamma^{*}-\theta_{r}\right)
\end{aligned}
$$

Here, the cornering power of the front and rear wheels, $K_{F}$ and $K_{R}$, are obtained from the tangent at the origin for the plot of side-slip angle verses cornering force in the nonlinear vehicle model

\subsection{Nonlinear dynamic equation of motion}

\subsubsection{Vehicle dynamics}

Figure 2 depicts the force vector used to construct the dynamic equations of motion for the vehicle and for the yaw rotational speed based on four-wheel drive and four-wheel steering. In the nonlinear model, there are no assumptions made that necessitates us to calculate the dynamic equations for longitude and latitude velocities, including the coupling of the yaw rotation speed with the velocities.

$$
\begin{aligned}
\mathrm{m}\left(\frac{d u}{d t}-v \gamma\right)= & \left(X_{F R}+X_{F L}\right) \cos \theta_{F}+\left(X_{R R}+X_{R L}\right) \cos \theta_{R}-\left(Y_{F R}+Y_{F L}\right) \sin \theta_{F} \\
& -\left(Y_{R R}+Y_{R L}\right) \sin \theta_{R} \\
\mathrm{~m}\left(\frac{d v}{d t}+u \gamma\right)= & \left(X_{F R}+X_{F L}\right) \sin \theta_{F}+\left(X_{R R}+X_{R L}\right) \sin \theta_{R}+\left(Y_{F R}+Y_{F L}\right) \cos \theta_{F} \\
& +\left(Y_{R R}+Y_{R L}\right) \cos \theta_{R}+G
\end{aligned}
$$




$$
\begin{aligned}
\mathrm{I} \frac{d \gamma}{d t}=l_{F}\left[\left(X_{F R}+\right.\right. & \left.\left.X_{F L}\right) \sin \theta_{F}+\left(Y_{F R}+Y_{F L}\right) \cos \theta_{F}\right] \\
& +l_{R}\left[\left(X_{R R}+X_{R L}\right) \sin \theta_{R}+\left(Y_{R R}+Y_{R L}\right) \cos \theta_{R}\right] \\
& +\frac{d_{F}}{2}\left[\left(X_{F R}+X_{F L}\right) \cos \theta_{F}+\left(Y_{F R}+Y_{F L}\right) \sin \theta_{F}\right] \\
& +\frac{d_{R}}{2}\left[\left(X_{R R}+X_{R L}\right) \cos \theta_{R}+\left(Y_{R R}+Y_{R L}\right) \sin \theta_{R}\right]+l_{d} G_{d}
\end{aligned}
$$

\subsubsection{Tire characteristics}

In the simulation, the wheels are modelled based on a brush tire model. Thus, in constructing the equations for friction and lateral forces, the slip ratio, tire side-slip angle, and weight distribution is taken into consideration. The model's deformation of the tire tread rubber is also used to derive as the following equations,

When $\xi_{\rho} \geq 0$,

$$
\begin{gathered}
X=-K_{\rho} \rho \xi_{p}{ }^{2}-6 \mu W_{z} \frac{\rho}{\lambda}\left(\frac{1}{6}-\frac{1}{2} \xi_{p}{ }^{2}+\frac{1}{3} \xi_{p}^{3}\right) \\
Y=-K_{\beta}(1+\rho) \tan \beta \xi_{p}{ }^{2}-6 \mu W_{z}\left(\frac{K_{\beta} \tan \beta(1+\rho)}{K_{\rho} \lambda}\right)\left(\frac{1}{6}-\frac{1}{2} \xi_{p}{ }^{2}+\frac{1}{3} \xi_{p}{ }^{3}\right)
\end{gathered}
$$

else,

$$
\begin{gathered}
X=-\mu W_{z} \frac{\rho}{\lambda} \\
Y=-\mu W_{z}\left(\frac{K_{\beta} \tan \beta(1+\rho)}{K_{\rho} \lambda}\right)
\end{gathered}
$$

Where,

$$
\xi_{p}=1-\frac{K_{\rho} \lambda}{3 \mu W_{z}(1-\rho)}, \quad \lambda=\sqrt{\rho^{2}+\left(\frac{K_{\beta}}{K_{\rho}}\right)^{2} \tan ^{2} \beta}, \quad K_{\rho}=\frac{b l_{T}{ }^{2}}{2} K_{x}, \quad K_{\beta}=\frac{b l_{T}^{2}}{2} K_{y}
$$

The equation for side-slip angle for four wheel steering tire is given as follows;

$$
\begin{aligned}
& \beta_{F R}=\tan ^{-1}\left(\frac{v+l_{F} \gamma}{u+d_{F} \gamma / 2}\right)-\theta_{F} \\
& \beta_{F L}=\tan ^{-1}\left(\frac{v+l_{F} \gamma}{u-d_{F} \gamma / 2}\right)-\theta_{F} \\
& \beta_{R R}=\tan ^{-1}\left(\frac{v-l_{R} \gamma}{u+d_{R} \gamma / 2}\right)-\theta_{R} \\
& \beta_{R L}=\tan ^{-1}\left(\frac{v-l_{R} \gamma}{u-d_{R} \gamma / 2}\right)-\theta_{R}
\end{aligned}
$$

The slip ratio $\rho$ can be represented in terms of the traction between the road and the tire surface, which is defined as;

$$
\rho=\frac{r \omega-u}{r \omega}
$$


Then the coefficient of friction $\mu$ can be approximated by the following equation;

$$
\mu=-1.10 \mathrm{k} \times\left(e^{35 \rho}-e^{0.35 \rho}\right)
$$

where the road coefficient $\mathrm{k}$ is set to 0.8 for normal dry asphalt road.

\section{Simulation Procedures}

The first stage of the simulation is to compare the vehicle motion with nonlinear and linear dynamic equation of motion of the modelled electric vehicle. The vehicle speed $V$ is set to gradual increase at start until it reached to constant speed at $10 \mathrm{~km} / \mathrm{h}$ with the front wheels' steer angle $\theta_{F}=5^{\circ}$ that initiated at time period of $\mathrm{t}=10 \mathrm{~s}$. The time frame is set to $\mathrm{t}=40 \mathrm{~s}$. This procedure is repeated for vehicle speed $V=20,30,40$ and $50 \mathrm{~km} / \mathrm{h}$, respectively.

The second stage of the simulation is to determine the characteristics of the four-wheel steering during steady state cornering. The parameters are the same as the first stage is term of the vehicle velocity $\mathrm{V}=10 \mathrm{~km} / \mathrm{h}$ and the front wheels' steer angle. In addition, the rear wheels' steer angle $\theta_{R}=\theta_{F}=5^{\circ}$ that initiated at time period of $t=10 \mathrm{~s}$ for the parallel steering mode. Then, this procedure is repeated for the rear wheels' steer angle $\theta_{R}=-\theta_{F}=-5^{\circ}$ for opposite steering mode.

\section{Result and Discussion}

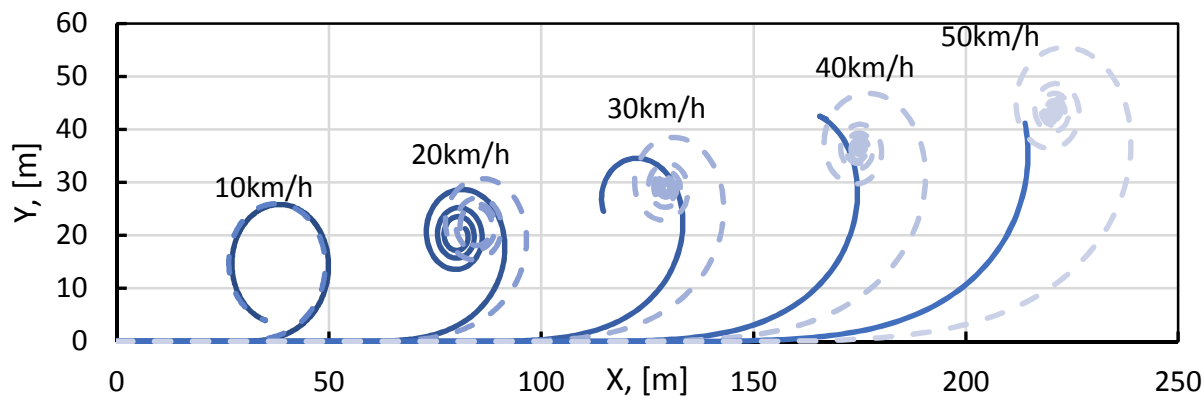

Fig.3 Simulation trajectory of the modelled electric vehicle with nonlinear and linear dynamic equation of motion during cornering at various speeds.

Figure 3 shows the trajectory of the simulated electric vehicle during cornering at various speed of $V=10,20,30,40$ and $50 \mathrm{~km} / \mathrm{h}$ at constant front wheels' steer angle of $\theta_{F}=5^{\circ}$. The solid lines and dash lines represent vehicle model with nonlinear and linear dynamic equation of motion, respectively. When the vehicle speed is at $10 \mathrm{~km} / \mathrm{h}$, the trajectory of the vehicle for nonlinear and linear are almost similar. However, the variant for both models can be clearly observed as the speed increased and all shows an oversteer characteristic based on the decreasing turning radius in the trajectory. In the case of linear model, a uniform pattern where the spiral of the cornering trajectory becomes smaller with vehicle speed. In the case of nonlinear model, the spiral can only be observed at vehicle speed $20 \mathrm{~km} / \mathrm{h}$. This is because during velocity $30 \mathrm{~km} / \mathrm{h}$ to $50 \mathrm{~km} / \mathrm{h}$, the simulation stopped at a certain period of time when it detects that the longitudinal speed $u$ has reached $0 \mathrm{~km} / \mathrm{h}$. Even though the vehicle speed is constant, the longitudinal speed $u$ can decrease while the lateral speed $v$ increase.

Figure 4 shows the yaw rotational speed of the simulated electric vehicle during cornering. When the vehicle speed is at $10 \mathrm{~km} / \mathrm{h}$, both nonlinear and linear model show a constant value at $0.244 \mathrm{rad} / \mathrm{s}$ that demonstrate a steady state cornering. However, the yaw rotational speed increase to an infinite value for the constant vehicle velocity $20 \mathrm{~km} / \mathrm{h}, 30$ 
$\mathrm{km} / \mathrm{h} 40 \mathrm{~km} / \mathrm{h}$ and $50 \mathrm{~km} / \mathrm{h}$. This result further prove that the vehicle indicated an oversteer characteristic.
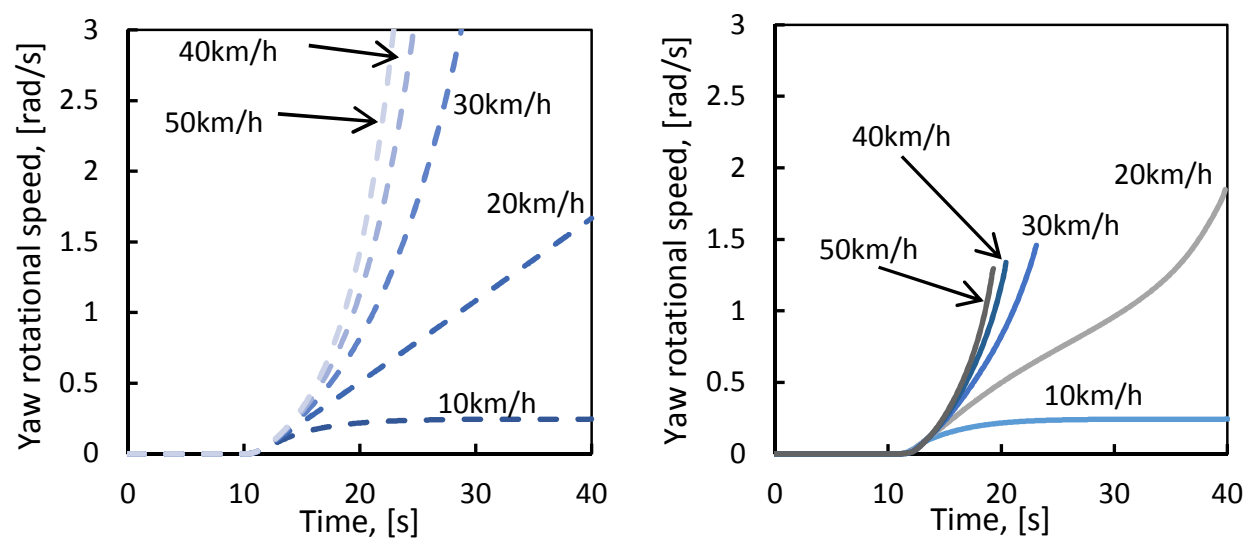

Fig.4 (left side) Yaw rotational speed of the modelled electric vehicle with linear dynamic equation of motion during cornering at various speeds. (right side) Yaw rotational speed of the modelled electric vehicle with nonlinear dynamic equation of motion during cornering at various speeds.

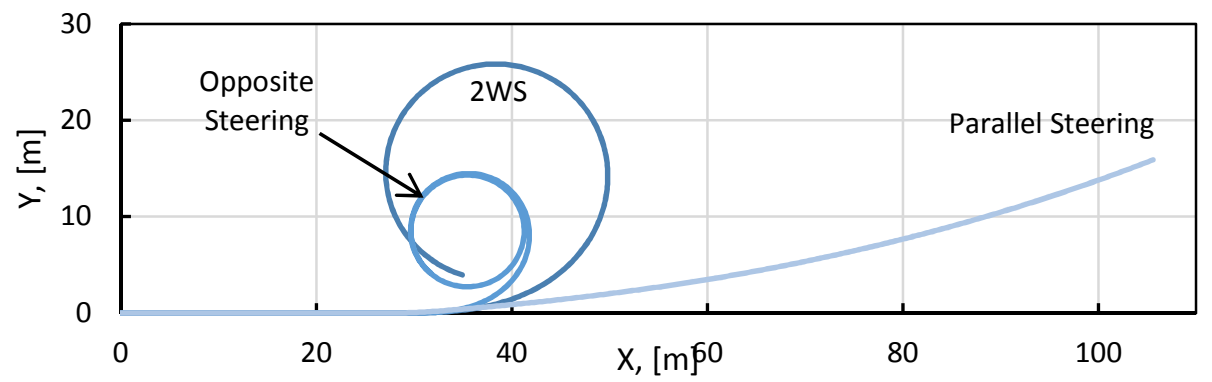

Fig.5 Simulation trajectory of the modelled electric vehicle during steady state cornering for $2 \mathrm{WS}$, opposite steering mode and parallel steering mode.

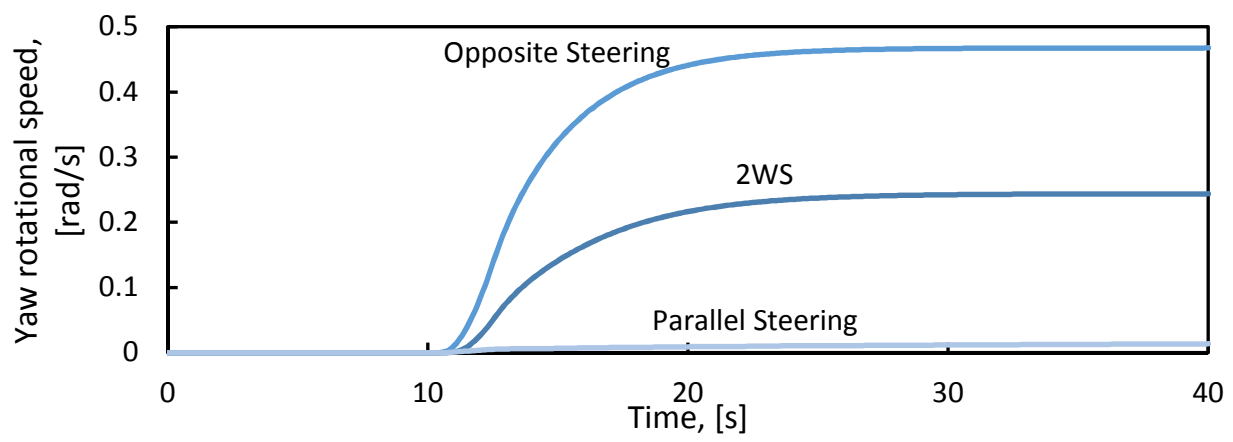

Fig.6 Yaw rotational speed of the modelled electric vehicle during steady state cornering for 2WS, opposite steering mode and parallel steering mode.

Based on the first stage of the simulation, cornering with four-wheel steering was simulated at $10 \mathrm{~km} / \mathrm{h}$. Figure 5 shows the trajectory of the modelled electric vehicle during steady state cornering for $2 \mathrm{WS}$, opposite steering mode and parallel steering mode. The trajectory of the opposite steering mode appears to have a smaller turning radius than the $2 \mathrm{WS}$ while the trajectory of the parallel steering mode demonstrates a less cornering effect and nearly having a translation motion only. In order to explain these occurrences, we can observe the yaw rotational speed of the simulated vehicle in Figure 6. When the rear wheels' steer angle is in the opposite direction to the front wheels, the yaw rotational speed will 
increase. In the simulation, the ratio of the rear to front wheels' angle is -1 which produce nearly twofold the yaw rotational speed of the $2 \mathrm{WS}$ from $0.244 \mathrm{rad} / \mathrm{s}$ to $0.467 \mathrm{rad} / \mathrm{s}$. On the contrary, the parallel steering mode produce almost no yaw rotational speed at $0.075 \mathrm{rad} / \mathrm{s}$.

\section{Conclusion}

A study on the vehicle dynamics of an electric vehicle with 4WD and 4WS based on nonlinear model was performed by numerical simulation. In the first stage of this study, where we analysed the variance of a linear model and nonlinear model of an electric vehicle during cornering, the results show that during low speed cornering at $10 \mathrm{~km} / \mathrm{h}$, the linear and nonlinear model produced similar steady state cornering based on the trajectory and yaw rotational speed. However, the variants of linear and nonlinear started to appear as the vehicle speed increase. In the second stage of the study, a numerical simulation was performed to analyse the characteristics of the 4WD and 4WS electric vehicle during cornering at constant speed and at a constant front wheel steer angle. A passive control of the rear wheels' steer angle was implement in the simulation. The results show that the parallel steering mode decreased the yaw rotational speed which broaden the trajectory of the cornering, while the opposite steering mode increased the yaw rotational speed that led to a tighter trajectory during cornering.

This research was supported by Automotive Engineering Centre (AEC), Faculty of Mechanical Engineering, Universiti Malaysia Pahang (RDU1703217).

\section{References}

1. F. Du, J. S. Li, L. Li, D. H. Si, Robust Control Study For Four-Wheel Active Steering Vehicle, Proceedings - International Conference on Electrical and Control Engineering, ICECE 2010, pp. 1830-1833, (2010).

2. N. Hamzah, M. K. Aripin, Y. M. Sam, H. Selamat, M. F Ismail, Yaw Stability Improvement For Four-Wheel Active Steering Vehicle Using Sliding Mode Control, 2012 IEEE 8th International Colloquium on Signal Processing and Its Applications, pp.127132, (2012).

3. J. Tian, N. Chen, J. Yang, L. Wang, Fractional Order Sliding Model Control Of Active Four-Wheel Steering Vehicles, ICFDA'14 International Conference on Fractional Differentiation and Its Applications 2014, (2014).

4. Ssu-Hsin Yu, John J.Moskwa, Journal of Dynamic Systems, Measurement, and Control ,116, pp.659-667, (1994),

5. S. Sano, Y. Furukawa and S. Shiraishi, Four Wheel Steering System with Rear Wheel Steer Angle Control as a Function of Steering Wheel Angle, SAE World Congress and Exhibition, Detroit, MI, USA, SAE Paper no.860625,

6. M. Abe, Vehicle Handling Dynamics: Theory and Application, Vehicle Handling Dynamics: Theory and Application, First Edition (Butterworth-Heinemann, 2009). 